\title{
Predictors of cardiac benefits of renal artery stenting from a multicentre retrospective registry
}

\author{
Yuri Nakajima, ${ }^{1}$ Osami Kawarada, ${ }^{1,2}$ Akihiro Higashimori, ${ }^{2}$ Yoshiaki Yokoi, ${ }^{2}$ \\ Kan Zen, ${ }^{3}$ Hitoshi Anzai, ${ }^{4}$ Hideki Doi, ${ }^{5}$ Seiji Hokimoto, ${ }^{6}$ Shigenori Ito, ${ }^{7}$ Taku Kato, ${ }^{8}$ \\ Teruyoshi Kume, ${ }^{9}$ Yoshiaki Shintani, ${ }^{10}$ Shuzou Tanimoto, ${ }^{11}$ Yoshinori Tsubakimoto, ${ }^{12}$ \\ Makoto Utsunomiya, ${ }_{1}^{13}$ Kunihiro Nishimura, ${ }^{14}$ Satoshi Yasuda ${ }^{1}$
}

For numbered affiliations see end of article.

\section{Correspondence to}

Dr Osami Kawarada,

Department of Cardiovascular Medicine, National Cerebral and Cardiovascular Center, 5-7-1 Fujishiro-dai, Suita, Osaka 5658565, Japan; kawarada90@ hotmail.com

Received 5 June 2017 Revised 18 August 2017 Accepted 19 August 2017

SLinked

- http://dx.doi.org/10.1136/ heartasia-2017-010951

CrossMark

To cite: Nakajima Y, Kawarada O, Higashimori A, et al. Heart Asia

2017:9:1-5. doi:10.1136/

heartasia-2017-010934

\section{ABSTRACT}

Objectives There have been limited data regarding the prediction of cardiac benefits after renal artery stenting for patients with atherosclerotic renal artery disease (ARAD). The aim of this multicentre retrospective study was to identify clinical or echocardiographic factors associated with improvements of cardiac symptoms after renal artery stenting.

Methods We enrolled 58 patients with de novo ARAD undergoing successful renal artery stenting for heart failure, angina or both between January 2000 and August 2015 at 13 hospitals.

Results Improvement of cardiac symptoms was observed in $86.2 \%$ of patients during a mean followup of $6.0 \pm 2.7$ months. Responders demonstrated significantly lower New York Heart Association functional class, higher estimated glomerular filtration rate, lower serum creatinine and lower interventricular septal wall thickness (IVS), lower left ventricular mass index, lower left atrial dimension and lower E-velocity than non-responders. Backward stepwise multivariate analysis identified IVS as an independent predictor of improvement of cardiac symptoms (OR 0.451, 95\% Cl 0.209 to $0.976 ; p=0.043)$. According to receiver operating characteristic curve analysis, an IVS cut-off of $11.9 \mathrm{~mm}$ provided the best predictive value, with sensitivity of $71.4 \%$, specificity of $75.5 \%$ and accuracy of $73.5 \%$. The positive predictive value was $74.5 \%$ and the negative predictive value was $72.5 \%$.

Conclusions This multicentre retrospective study shows that the echocardiographic index of IVS is an independent predictor for improvement of cardiac symptoms after renal artery stenting.

\section{INTRODUCTION}

Cardiac disorders related to atherosclerotic renal artery disease (ARAD) are an important clinical entity in the field of cardiovascular medicine. ${ }^{1-3}$ Although recent prospective randomised studies failed to demonstrate the advantage of renal artery stenting over medical therapy in terms of controlling blood pressure and renal function and preventing cardiovascular events, it is not possible to enrol patients with fluctuating clinical conditions, who are most likely to benefit from renal artery stenting, in randomised studies. ${ }^{4}$ In the most updated statement, ${ }^{5}$ cardiac disorders such as heart failure and unstable angina are categorised as appropriate clinical indications for renal revascularisation. The aim of this study was therefore to identify predictors of cardiac benefits after renal artery stenting.

\section{METHODS}

The PREMIERE registry (Predictors of Cardiac Benefits of Renal Artery Stenting from A Multicenter Retrospective Registry) is a multicentre retrospective registry consisting of patients with de novo ARAD who underwent successful renal artery stenting for the control of heart failure, angina or both between January 2000 and August 2015 at 13 vascular centres in Japan. The diagnosis of heart failure and angina pectoris was based on the physician's clinical judgement with reference to criteria in the Framingham study and the previous guidelines. ${ }^{6}$ Renal artery stenting was performed according to standard procedures. All patients received dual antiplatelet therapy before the procedure. The choice of stent type and route of vascular access was left to the individual operator's discretion. Follow-up examinations, which included evaluation of clinical symptoms and blood pressure, blood testing and echocardiography, were conducted before discharge and within 12 months.

Subjects were categorised as responders or non-responders. Responders were defined as patients demonstrating improved cardiac symptoms during the follow-up period, such as achieving a lower New York Heart Association (NYHA) functional class and no re-hospitalisation for heart failure, angina or both. Non-responders were defined as patients showing no improvement of cardiac symptoms. The institutional review boards in each institute approved the study.

\section{Clinical and echocardiographic parameters}

The preinterventional clinical and echocardiographic variables of enrolled patients were retrospectively reviewed at each institution. Clinical variables including risk factors, concomitant vascular disease, NYHA class, blood pressure, renal function and B-type natriuretic peptide (BNP) were abstracted. The echocardiographic data included the left ventricular dimension in diastole and systole, interventricular septal wall thickness (IVS), posterior wall thickness (PWT), left ventricular mass index (LVMI), left atrial dimension (LAD), left ventricular ejection fraction, peak early diastolic mitral inflow velocity (E-velocity), peak early diastolic mitral annular 
velocity in the septum ( $\mathrm{e}^{\prime}$-velocity), and E/e' ratio. LVMI was calculated as left ventricular mass (LVM)/body surface area (BSA). LVM was calculated using the modified Devereux formula. ${ }^{8}$ BSA was calculated using the formula of Du Bois and Du Bois. ${ }^{9}$

\section{Sample size determination}

Assumptions for sample size calculations were based on previous studies of the effect of renal artery stenting on angina or heart failure. ${ }^{10} 11$ Forty patients were needed with the assumption of a $10 \%$ drop out. This sample size would allow $75 \%$ effectiveness of renal artery stenting to be detected by a two-sided exact test for one proportion with power 0.9 and $\alpha=0.05$ using PASS 14 (NCSS, Kaysville, Utah, USA).

\section{Statistical analysis}

Data are expressed as mean \pm SD or number (\%). Categorical data were compared using the $\chi^{2}$ test or Fisher exact test. Differences between the two independent cohorts were evaluated with the Student t-test or Welch t-test for parametric continuous variables or the Mann-Whitney test for non-parametric continuous variables. Normality for distributions of continuous variables was determined using the Shapiro-Wilk test. Changes in blood pressure and renal function were assessed using one-way repeated analysis of variance with Bonferroni's post hoc test or Friedman test. All variables tested in univariate analysis with $\mathrm{p}<0.15$ were included in a multivariate logistic regression analysis with backward stepwise selection. Considering the correlations between estimated glomerular filtration rate (eGFR) and serum creatinine and between E-velocity and $\mathrm{E} / \mathrm{e}^{\prime}$, serum creatinine and E-velocity were excluded from the multivariate model to avoid multicollinearity using variation inflation factors. $\mathrm{p}<0.05$ was considered significant. Statistical analysis was performed with SPSS Version 22 (SPSS, Chicago, Illinois, USA).

\section{RESULTS}

\section{Clinical and echocardiographic characteristics}

Fifty-eight patients were enrolled in the registry. The baseline clinical characteristics of the study subjects are shown in table 1 . The average age was $74.4 \pm 10.2$ years (range $47-90$ years). The most common atherosclerotic risk factor was hypertension. As for concomitant atherosclerotic disease, coronary artery disease was most frequent, followed by peripheral artery disease. Bilateral ARAD undergoing bilateral renal artery stenting was observed in $20.7 \%$. Baseline echocardiographic characteristics are shown in table 2. The ejection fraction was $54.3 \pm 13.7 \%$ and E-velocity/e'-velocity in the septal wall was $18.6 \pm 8.1$, suggesting preservation of left ventricular systolic function and impairment of left ventricular diastolic function.

\section{Responders versus non-responders}

During the mean follow-up time of $6.0 \pm 2.7$ months (range 1-12 months), systolic and diastolic blood pressure significantly decreased whereas the number of antihypertensive agents and renal function remained unchanged (table 3 ). With respect to cardiac benefits, 50 patients $(86.2 \%)$ were responders and 8 $(13.8 \%)$ were non-responders. Comparisons of clinical variables between responders and non-responders are shown in table 4. Responders demonstrated significantly lower NYHA functional class, higher eGFR and lower serum creatinine than non-responders. Comparisons of echocardiographic variables

\begin{tabular}{ll}
\hline Table 1 & Baseline clinical characteristics \\
\hline Patients, $\mathrm{n}$ & 58 \\
\hline Age, years & $74.4 \pm 10.2$ \\
\hline Men, $\mathrm{n}(\%)$ & $33(56.9 \%)$ \\
\hline Hypertension, $\mathrm{n}(\%)$ & $57(98.3 \%)$ \\
\hline Diabetes mellitus, $\mathrm{n}(\%)$ & $21(36.2 \%)$ \\
\hline Dyslipidaemia, $\mathrm{n}(\%)$ & $41(70.7 \%)$ \\
\hline History of smoking, $\mathrm{n}(\%)$ & $28(48.3 \%)$ \\
\hline Coronary artery disease, $\mathrm{n}(\%)$ & $45(77.6 \%)$ \\
\hline Peripheral artery disease, $\mathrm{n}(\%)$ & $23(39.7 \%)$ \\
\hline Cerebrovascular disease, $\mathrm{n}(\%)$ & $10(17.2 \%)$ \\
\hline Bilateral disease, $\mathrm{n}(\%)$ & $12(20.7 \%)$ \\
\hline NYHA functional class & $2.5 \pm 1.0$ \\
\hline Antihypertensive medicine, $\mathrm{n}$ & $2.8 \pm 1.3$ \\
\hline Systolic blood pressure $(\mathrm{mm} \mathrm{Hg})$ & $146.5 \pm 23.5$ \\
\hline Diastolic blood pressure $(\mathrm{mm} \mathrm{Hg})$ & $73.4 \pm 13.2$ \\
\hline Serum creatinine $(\mathrm{mg} / \mathrm{dL})$ & $1.38 \pm 0.72$ \\
\hline eGFR (mL/min/1.73 m²) & $43.4 \pm 19.9$ \\
\hline BNP & $451.9 \pm 536.5$ \\
\hline log BNP & $2.36 \pm 0.55$ \\
\hline BNP B-type natiun & \\
\hline
\end{tabular}

BNP, B-type natriuretic peptide; eGFR, estimated glomerular filtration rate; NYHA, New York Heart Association.

between responders and non-responders are shown in table 5 . Responders showed significantly lower IVS, LVMI, LAD and E-velocity than responders. The IVS:PWT ratio was numerically lower in responders than in non-responders $(0.99$ vs $1.10, p=0.221$ ), but the difference was not significant. Backward stepwise multivariate analysis using these variables with $\mathrm{p}<0.15$ (hypertension, diabetes mellitus, NYHA class, eGFR, IVS, LVMI, LAD and E/e') identified a cardiac parameter, IVS, as the only independent predictor of improvement in cardiac symptoms (OR $0.451,95 \%$ CI 0.209 to $0.976 ; \mathrm{p}=0.043$ ), and both eGFR $(p=0.070)$ and $E / e^{\prime}(p=0.097)$ were not significant in the final step of the analysis.

\section{Receiver operating characteristic curve analysis}

A receiver operating characteristic (ROC) curve, illustrating the accuracy of IVS for predicting the improvement of cardiac symptoms, is shown in figure 1 . The area under the ROC curve was 0.819 (95\% CI 0.683 to $0.956 ; p=0.007)$. An IVS cut-off value of $11.9 \mathrm{~mm}$ provided the best predictive value with sensitivity of $71.4 \%$, specificity of $75.5 \%$ and accuracy of $73.5 \%$. The positive predictive value was $74.5 \%$ and the negative predictive value was $72.5 \%$.

\begin{tabular}{ll}
\hline Table 2 & Baseline echocardiographic characteristics \\
\hline Left ventricular dimension in diastole $(\mathrm{mm})$ & $48.6 \pm 8.2$ \\
\hline Left ventricular dimension in systole $(\mathrm{mm})$ & $34.6 \pm 9.6$ \\
\hline Interventricular septal wall thickness $(\mathrm{mm})$ & $10.8 \pm 1.9$ \\
\hline Posterior wall thickness $(\mathrm{mm})$ & $10.9 \pm 1.6$ \\
Interventricular septal wall thickness/posterior wall thickness & $1.00 \pm 0.14$ \\
Left ventricular mass index $\left(\mathrm{g} / \mathrm{m}^{2}\right)$ & $132 \pm 37$ \\
Left atrial dimension $(\mathrm{mm})$ & $40.1 \pm 8.2$ \\
Left ventricular ejection fraction $(\%)$ & $54.3 \pm 13.7$ \\
E-velocity (cm/s) & $84.4 \pm 29.9$ \\
\hline $\mathrm{e}^{\prime}$-velocity $(\mathrm{cm} / \mathrm{s})$ & $4.7 \pm 1.4$ \\
\hline E/e' & $18.6 \pm 8.1$ \\
\hline
\end{tabular}

Nakajima Y, et al. Heart Asia 2017;9:1-5. doi:10.1136/heartasia-2017-010934 
Table 3 Blood pressure and renal function before and after renal artery stenting

\begin{tabular}{llllc}
\hline & Pre & Post & Follow-up & p Value \\
\hline $\begin{array}{l}\text { Systolic blood pressure } \\
\text { (mm Hg) }\end{array}$ & $148 \pm 23$ & $131 \pm 19^{*}$ & $132 \pm 19^{*}$ & $<0.001$ \\
$\begin{array}{l}\text { Diastolic blood pressure } \\
(\mathrm{mm} \mathrm{Hg})\end{array}$ & $75 \pm 13$ & $70 \pm 11$ & $68 \pm 11 \dagger$ & 0.03 \\
\hline No of antihypertensive agents & $2.7 \pm 1.3$ & $2.8 \pm 1.4$ & $2.7 \pm 1.3$ & 0.767 \\
\hline $\begin{array}{l}\text { Serum creatinine }(\mathrm{mg} / \mathrm{dL}) \\
\text { eGFR }\left(\mathrm{mL} / \mathrm{min} / 1.73 \mathrm{~m}^{2}\right)\end{array}$ & $1.36 \pm 0.71$ & $1.38 \pm 0.83$ & $1.38 \pm 0.71$ & 0.937 \\
\hline $\begin{array}{l}{ }^{*} \mathrm{p}<0.001 \text { versus pre. } \\
\mathrm{tp}<0.05 \text { versus pre. }\end{array}$ & $44 \pm 20$ & $45 \pm 21$ & $44 \pm 19$ & 0.864 \\
\hline
\end{tabular}

\section{DISCUSSION}

Given an increasing awareness of the relationship between ARAD and cardiac disorders and the effects of renal artery stenting on cardiac disorders, ${ }^{1}$ the present study investigated predictors of improvement of cardiac symptoms after renal artery stenting in patients with ARAD. The main findings of this study were as follows: (1) $86.2 \%$ of the patients were responders; (2) in univariate analysis, NYHA functional class, renal function (eGFR and serum creatinine) and four echocardiographic indices (IVS, LVMI, LAD and E-velocity) were significantly associated with improvement of cardiac symptoms; (3) multivariate analysis showed IVS to be the strongest independent predictor of improvement in cardiac symptoms; and (4) an IVS of $11.9 \mathrm{~mm}$ best predicted improvement of cardiac symptoms, with a sensitivity of $71.4 \%$, specificity of $75.5 \%$, and accuracy of $73.5 \%$ in ROC curve analysis.

There is discordance between the very high (>95\%) procedural success rate for renal revascularisation and the moderate $(60-70 \%)$ clinical response rate for the treatment of hypertension and renal failure. ${ }^{12}$ Previous investigators identified several predictors of improvement in blood pressure and renal function. Predictors of blood pressure improvement include baseline systolic or mean blood pressure, renal parenchymal thickness, number of antihypertensive agents, bilateral disease,

Table 4 Comparison of clinical variables between responders and non-responders

\begin{tabular}{lllc}
\hline & & $\begin{array}{l}\text { Non- } \\
\text { responders }\end{array}$ & \\
& Responders $(\mathrm{n}=50)$ & $\mathrm{n}=8)$ & $\mathrm{p}$ Value \\
\hline Age, years & $73.7 \pm 10.7$ & $79.1 \pm 4.3$ & 0.242 \\
\hline Men, $\mathrm{n}(\%)$ & $28(56.0 \%)$ & $6(62.5 \%)$ & 0.685 \\
\hline Hypertension, $\mathrm{n}(\%)$ & $50(100 \%)$ & $7(87.5 \%)$ & 0.138 \\
\hline Diabetes mellitus, $\mathrm{n}(\%)$ & $16(32.0 \%)$ & $5(62.5 \%)$ & 0.124 \\
\hline Dyslipidaemia, $\mathrm{n}(\%)$ & $34(68.0 \%)$ & $7(87.5 \%)$ & 0.415 \\
\hline History of smoking, $\mathrm{n}(\%)$ & $23(46.0 \%)$ & $5(62.5 \%)$ & 0.464 \\
\hline Coronary artery disease, $\mathrm{n}(\%)$ & $39(78.0 \%)$ & $6(75.0 \%)$ & 1 \\
\hline Peripheral artery disease, $\mathrm{n}(\%)$ & $19(38.0 \%)$ & $4(50.0 \%)$ & 0.7 \\
\hline Cerebrovascular disease, $\mathrm{n}(\%)$ & $8(16.0 \%)$ & $2(25.0 \%)$ & 0.419 \\
\hline Bilateral disease, $\mathrm{n}(\%)$ & $12(24.0 \%)$ & $0(0 \%)$ & 0.185 \\
\hline NYHA functional class & $2.4 \pm 0.9$ & $3.3 \pm 1.2$ & 0.035 \\
\hline Antihypertensive medicine, $\mathrm{n}$ & $2.7 \pm 1.3$ & $3.3 \pm 1.8$ & 0.322 \\
\hline Systolic blood pressure $(\mathrm{mm} \mathrm{Hg})$ & $146.3 \pm 24.9$ & $148.3 \pm 13.2$ & 0.827 \\
\hline Diastolic blood pressure $(\mathrm{mm} \mathrm{Hg})$ & $74.2 \pm 13.3$ & $68.5 \pm 12.0$ & 0.261 \\
\hline eGFR (mL/min/1.73 m²) & $46.3 \pm 19.5$ & $25.4 \pm 11.6$ & 0.005 \\
\hline Serum creatinine (mg/dL) & $1.26 \pm 0.62$ & $2.17 \pm 0.86$ & $<0.001$ \\
\hline log BNP & $2.33 \pm 0.56$ & $2.68 \pm 0.35$ & 0.178 \\
\hline
\end{tabular}

Table 5 Comparison of echocardiographic variables between responders and non-responders

\begin{tabular}{|c|c|c|c|}
\hline & $\begin{array}{l}\text { Responders } \\
(n=50)\end{array}$ & $\begin{array}{l}\text { Non-responders } \\
(n=8)\end{array}$ & $\mathrm{p}$ Value \\
\hline $\begin{array}{l}\text { Left ventricular dimension in } \\
\text { diastole (mm) }\end{array}$ & $48.0 \pm 8.5$ & $52.4 \pm 5.2$ & 0.186 \\
\hline $\begin{array}{l}\text { Left ventricular dimension in } \\
\text { systole }(\mathrm{mm})\end{array}$ & $34.4 \pm 10.1$ & $36.3 \pm 5.0$ & 0.436 \\
\hline $\begin{array}{l}\text { Interventricular septal wall } \\
\text { thickness (mm) }\end{array}$ & $10.6 \pm 1.9$ & $12.4 \pm 1.3$ & 0.005 \\
\hline Posterior wall thickness (mm) & $10.8 \pm 1.6$ & $11.4 \pm 1.5$ & 0.271 \\
\hline $\begin{array}{l}\text { Interventricular septal wall } \\
\text { thickness/posterior wall } \\
\text { thickness }\end{array}$ & $0.99 \pm 0.13$ & $1.10 \pm 0.19$ & 0.221 \\
\hline $\begin{array}{l}\text { Left ventricular mass index }(\mathrm{g} / \\
\left.\mathrm{m}^{2}\right)\end{array}$ & $128.2 \pm 36.8$ & $159.0 \pm 28.5$ & 0.025 \\
\hline Left atrial dimension (mm) & $39.5 \pm 8.4$ & $45.0 \pm 3.9$ & 0.04 \\
\hline $\begin{array}{l}\text { Left ventricular ejection fraction } \\
(\%)\end{array}$ & $54.1 \pm 14.2$ & $55.7 \pm 9.9$ & 0.892 \\
\hline E-velocity $(\mathrm{cm} / \mathrm{s})$ & $79.7 \pm 25.3$ & $115.7 \pm 40.8$ & 0.002 \\
\hline $\mathrm{e}^{\prime}$-velocity (cm/s) & $4.7 \pm 1.4$ & $4.7 \pm 1.4$ & 0.815 \\
\hline$E / e^{\prime}$ & $17.6 \pm 7.2$ & $24.0 \pm 11.1$ & 0.125 \\
\hline
\end{tabular}

renal fractional flow reserve, BNP, translesional pressure gradient and renal frame count. Predictors of renal function improvement include baseline serum creatinine, percentage diameter stenosis, rapid increase in serum creatinine, bilateral revascularisation and left ventricular function. ${ }^{13-24}$ These findings suggest that factors predicting a better response after renal revascularisation are distinct for different target endpoints. However, there is a paucity of data regarding the prediction of cardiac benefits after renal artery stenting.

Sutters et al and Pickering et al initially reported the cardiac benefits of balloon renal angioplasty approximately three decades ago. ${ }^{25} 26$ In the stent era, Khosla et al reported that cardiac symptoms improved by 24 hours after renal artery stenting in $88 \%$ of patients, and clinical benefits

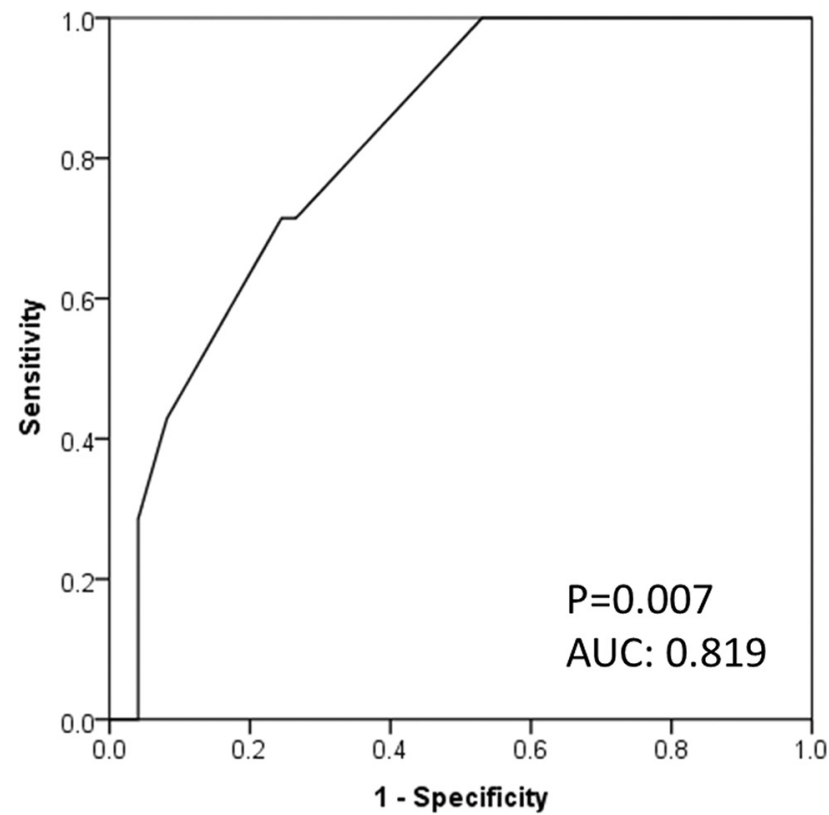

Figure 1 Receiver operator characteristic curve for interventricular septal wall thickness (IVS); a cut-off of $11.9 \mathrm{~mm}$ best predicted the improvement of cardiac symptoms. 
were sustained in $72.5 \%$ of patients with a mean follow-up of $253 \pm 192$ days. ${ }^{10}$ Subsequent single-centre retrospective studies reported improvements in heart failure and angina symptoms, number of hospitalisations for heart failure, proportion hospitalised for heart failure and time to hospitalisation for heart failure after renal artery stenting. ${ }^{11} 2728$ The response rate of renal artery stenting for cardiac disorders was in the range of $72.0 \%$ to $88.0 \%$. $^{2} 10112728$ In the present multicentre retrospective study, $86.2 \%$ of patients experienced improvement of cardiac symptoms during the mean follow-up period of $6.0 \pm 2.7$ months, a finding consistent with the previous studies.

The present study, which employed cardiac symptoms as an endpoint in univariate analysis, found that NYHA functional class, renal function and echocardiographic indices such as IVS, LVMI, LAD and E-velocity were associated with improvement of cardiac symptoms. Furthermore, stepwise multivariate analysis found that IVS was an independent predictor (OR 0.451 , 95\% CI 0.209 to 0.976 ; $\mathrm{p}=0.043$ ) of improvement of cardiac symptoms and eGFR yielded a trend $(p=0.07)$ towards less improvement of cardiac symptoms. ROC curve analysis showed that an IVS cut-off of $11.9 \mathrm{~mm}$ was optimal in terms of sensitivity and specificity for predicting improvement of cardiac symptoms. According to the previous studies regarding cardiac morphology, ${ }^{29-31}$ ARAD affected the heart more adversely than essential hypertension, and hypertrophic responses differed between patients with and without ARAD. Losito et al reported that left ventricular hypertrophy was present more often in patients with ARAD than in those with essential hypertension (32.6\% vs $10.8 \%, \mathrm{p}=0.02) .{ }^{30}$ According to Wright et al, patients with ARAD have a higher prevalence of left ventricular hypertrophy $(78.5 \%$ vs $46.0 \%, p<0.001)$ and higher LVMI $\left(183 \pm 74\right.$ vs $\left.116 \pm 33 \mathrm{~g} / \mathrm{m}^{2}, \mathrm{p}<0.001\right)$ than controls. ${ }^{31}$ Of great interest, Vensel et al found that patients with ARAD had greater disproportionate hypertrophy of the IVS than those with essential hypertension. ${ }^{29}$ In the context of the cardiac geometric pattern in patients with ARAD, recent studies reported that eccentric hypertrophy was associated with renal dysfunction. ${ }^{32} 33$ Thus, greater IVS might imply a more advanced disorder-related ARAD in the heart and kidney and suggests less improvement of cardiac symptoms after renal revascularisation. The present finding that responders had less severe cardiac structural changes and better renal function indicates the clinical relevance of renal revascularisation at the early stage rather than the advanced stage, and therefore further studies are needed.

Some limitations of this study should be taken into consideration. First, our sample size was relatively small and the study was retrospective in nature. Second, the potentially disparate quality of echocardiographic evaluation might have varied among invited hospitals, depending on the machine used and on the skills of the sonographers. Third, in cases of ARAD patients with concomitant coronary artery disease, coronary revascularisation might have affected the outcomes. Finally, this study did not employ other metrics including renal duplex ultrasonography.

\section{CONCLUSIONS}

In this multicentre retrospective study, IVS was independently associated with improvement in cardiac symptoms after renal artery stenting although a large prospective study is needed to confirm the clinical significance of assessment of cardiac function in patients with ARAD.
Key messages

What is already known about this subject? According to the most updated statement, cardiac disorders are categorised as appropriate clinical indications for renal revascularisation. Also, the response rate of renal artery stenting for cardiac disorders is in the range of $72.0-88.0 \%$.

\section{What does this study add?}

This study reported the performance of echocardiographic indices for predicting cardiac benefits after renal artery stenting. An interventricular septal wall thickness cut-off of $11.9 \mathrm{~mm}$ provided the best predictive value, with a sensitivity of $71.4 \%$, specificity of $75.5 \%$ and accuracy of $73.5 \%$. The positive predictive value was $74.5 \%$ and the negative predictive value was $72.5 \%$.

\section{How might this impact on clinical practice?}

This might increase awareness of the importance of cardiac function assessment in the management of atherosclerotic renal artery disease.

\section{Author affiliations}

${ }^{1}$ Department of Cardiovascular Medicine, National Cerebral and Cardiovascular Center, Osaka, Japan

${ }^{2}$ Department of Cardiology, Kishiwada Tokushukai Hospital, Osaka, Japan

${ }^{3}$ Department of Cardiology, Omihachiman Community Medical Center, Shiga, Japan

${ }^{4}$ Department of Cardiology, Ota Memorial Hospital, Gunma, Japan

${ }^{5}$ Department of Cardiology, Kumamoto Rosai Hospital, Kumamoto, Japan

${ }^{6}$ Department of Cardiology, Kumamoto University Hospital, Kumamoto, Japan

${ }^{7}$ Department of Cardiology, Nagoya City East Medical Center, Aichi, Japan

${ }^{8}$ Department of Cardiology, Nantan General Hospital, Kyoto, Japan

${ }^{9}$ Department of Cardiology, Kawasaki Medical School Hospital, Okayama, Japan

${ }^{10}$ Department of Cardiology, Shin Koga Hospital, Fukuoka, Japan

${ }^{11}$ Department of Cardiology, Mitsui Memorial Hospital, Tokyo, Japan

${ }^{12}$ Department of Cardiology, Kyoto Second Red Cross Hospital, Kyoto, Japan

${ }^{13}$ Department of Cardiology, Tokyo Rosai Hospital, Tokyo, Japan

${ }^{14}$ Department of Statistics and Data Analysis, Center for Cerebral and Cardiovascular

Disease Information, National Cerebral and Cardiovascular Center, Osaka, Japan

Contributors Each author has contributed significantly to the submitted work. Conception and design or analysis and interpretation of data, or both: YN, OK. Drafting of the manuscript or revising it critically for important intellectual content: YN, OK, SY. Data collection: YN, OK, AH, YY, KZ, HA, HD, SH, SI, TKa, TKu, YS, ST, YT, MU. Sample size determination: KN.

Competing interests None declared.

Patient consent Not needed because this study is a retrospective observational study.

Ethics approval National Cerebral and Cardiovascular Center.

Provenance and peer review Not commissioned; externally peer reviewed.

(c) Article author(s) (or their employer(s) unless otherwise stated in the text of the article) 2017. All rights reserved. No commercial use is permitted unless otherwise expressly granted.

\section{REFERENCES}

1 Missouris CG, Belli AM, MacGregor GA. "Apparent" heart failure: a syndrome caused by renal artery stenoses. Heart 2000;83:152-5

2 Messerli FH, Bangalore S, Makani H, et al. Flash pulmonary oedema and bilateral renal artery stenosis: the Pickering syndrome. Eur Heart J 2011;32:2231-5.

3 Kawarada 0, Yasuda S, Noguchi T, et al. Renovascular heart failure: heart failure in patients with atherosclerotic renal artery disease. Cardiovasc Interv Ther 2016:31:171-82.

4 White CJ. The "chicken little" of renal stent trials: the CORAL trial in perspective. JACC Cardiovasc Interv 2014;7:111-3.

5 Parikh SA, Shishehbor MH, Gray BH, et al. SCAl expert consensus statement for renal artery stenting appropriate use. Catheter Cardiovasc Interv 2014;84:1163-71.

6 McKee PA, Castelli WP, McNamara PM, et al. The natural history of congestive heart failure: the Framingham study. N Engl J Med 1971;285:1441-6. 
7 King SB, Smith SC, Hirshfeld JW, et al. 2007 Focused Update of the ACC/AHA/SCAI 2005 Guideline Update for Percutaneous Coronary Intervention: a report of the American College of Cardiology/American Heart Association Task Force on Practice Guidelines: 2007 Writing Group to Review New Evidence and Update the ACC/AHA/ SCAI 2005 Guideline Update for Percutaneous Coronary Intervention, Writing on Behalf of the 2005 Writing Committee. Circulation 2008;117:261-95.

8 Devereux RB, Alonso DR, Lutas EM, et al. Echocardiographic assessment of left ventricular hypertrophy: comparison to necropsy findings. Am I Cardiol 1986;57:450-8.

9 Du Bois D, Du Bois EF. A formula to estimate the approximate surface area if height and weight be known. 1916. Nutrition 1989;5:303-11.

10 Khosla S, White CJ, Collins TJ, et al. Effects of renal artery stent implantation in patients with renovascular hypertension presenting with unstable angina or congestive heart failure. Am J Cardiol 1997;80:363-6.

11 Gray BH, Olin JW, Childs MB, et al. Clinical benefit of renal artery angioplasty with stenting for the control of recurrent and refractory congestive heart failure. Vasc Med 2002;7:275-9.

12 White CJ, Olin JW. Diagnosis and management of atherosclerotic renal artery stenosis: improving patient selection and outcomes. Nat Clin Pract Cardiovasc Med 2009;6:176-90.

13 Protasiewicz M, Kądziela J, Początek K, et al. Renal artery stenosis in patients with resistant hypertension. Am J Cardiol 2013;112:1417-20.

14 Zeller T, Frank U, Müller C, et al. Predictors of improved renal function after percutaneous stent-supported angioplasty of severe atherosclerotic ostial renal artery stenosis. Circulation 2003;108:2244-9.

15 Zeller T, Frank U, Müller C, et al. Stent-supported angioplasty of severe atherosclerotic renal artery stenosis preserves renal function and improves blood pressure control: long-term results from a prospective registry of 456 lesions. J Endovasc Ther 2004;11:95-106.

16 Rocha-Singh KJ, Mishkel GJ, Katholi RE, et al. Clinical predictors of improved longterm blood pressure control after successful stenting of hypertensive patients with obstructive renal artery atherosclerosis. Catheter Cardiovasc Interv 1999;47:167-72.

17 Burket MW, Cooper CJ, Kennedy DJ, et al. Renal artery angioplasty and stent placement: predictors of a favorable outcome. Am Heart J 2000;139:64-71.

18 Mitchell JA, Subramanian R, White CJ, et al. Predicting blood pressure improvement in hypertensive patients after renal artery stent placement: renal fractional flow reserve. Catheter Cardiovasc Interv 2007;69:685-9.

19 Silva JA, Chan AW, White CJ, et al. Elevated brain natriuretic peptide predicts blood pressure response after stent revascularization in patients with renal artery stenosis. Circulation 2005;111:328-33.
20 Leesar MA, Varma J, Shapira A, et al. Prediction of hypertension improvement after stenting of renal artery stenosis: comparative accuracy of translesional pressure gradients, intravascular ultrasound, and angiography. J Am Coll Cardiol 2009:53:2363-71.

21 Naghi J, Palakodeti S, Ang L, et al. Renal frame count: a measure of renal flow that predicts success of renal artery stenting in hypertensive patients. Catheter Cardiovasc Interv 2015;86:304-9.

22 Weinberg I, Keyes MJ, Giri J, et al. Blood pressure response to renal artery stenting in 901 patients from five prospective multicenter FDA-approved trials. Catheter Cardiovasc Interv 2014;83:603-9.

23 Ritchie J, Green D, Chrysochou C, et al. High-risk clinical presentations in atherosclerotic renovascular disease: prognosis and response to renal artery revascularization. Am J Kidney Dis 2014;63:186-97.

24 Jaff MR, Bates M, Sullivan T, et al. Significant reduction in systolic blood pressure following renal artery stenting in patients with uncontrolled hypertension: results from the HERCULES trial. Catheter Cardiovasc Interv 2012;80:343-50.

25 Sutters M, Al-Kutoubi MA, Mathias CJ, et al. Diuresis and syncope after renal angioplasty in a patient with one functioning kidney. Br Med J 1987;295:527-8.

26 Pickering TG, Herman L, Devereux RB, et al. Recurrent pulmonary oedema in hypertension due to bilateral renal artery stenosis: treatment by angioplasty or surgical revascularisation. Lancet 1988;2:551-2.

27 Bloch MJ, Trost DW, Pickering TG, et al. Prevention of recurrent pulmonary edema in patients with bilateral renovascular disease through renal artery stent placement. $A m$ $J$ Hypertens 1999;12:1-7.

28 Kane GC, Xu N, Mistrik E, et al. Renal artery revascularization improves heart failure control in patients with atherosclerotic renal artery stenosis. Nephrol Dial Transplant 2010;25:813-20.

29 Vensel LA, Devereux RB, Pickering TG, et al. Cardiac structure and function in renovascular hypertension produced by unilateral and bilateral renal artery stenosis. Am J Cardiol 1986;58:575-82.

30 Losito A, Fagugli RM, Zampi I, et al. Comparison of target organ damage in renovascular and essential hypertension. Am J Hypertens 1996;9:1062-7.

31 Wright JR, Shurrab AE, Cooper A, et al. Left ventricular morphology and function in patients with atherosclerotic renovascular disease. J Am Soc Nephrol 2005:16:2746-53.

32 Khangura KK, Eirin A, Kane GC, et al. Cardiac function in renovascular hypertensive patients with and without renal dysfunction. Am J Hypertens 2014;27:445-53.

33 Khan AR, Sheikh M, Kaw D, et al. Prevalence and factors associated with left ventricular remodeling in renal artery stenosis. J Am Soc Hypertens 2014;8:254-61. 